\title{
Presupuestos participativos e instituciones políticas locales. Posibles formas de articulación en casos de Argentina y Uruguay
}

\author{
Participatory budgets and local political institutions. \\ Possible forms of articulation in cases of \\ Argentina and Uruguay
}

MARIANO SUÁREZ ELÍAS (Universidad de la República, Sede Salto)

Artículo recibido: 22 de agosto de 2019

Solicitud de revisión: 23 de septiembre de 2019

Artículo aceptado: 20 de noviembre de 2019

Suárez, Mariano (2020). Presupuestos participativos e instituciones políticas locales. Posibles formas de articulación en casos de Argentina y Uruguay. Recerca. Revista de Pensament i Análisi, 25(1), pp. 111-134.

Resumen

El objetivo del artículo es analizar las formas de articulación entre el presupuesto participativo y las instituciones político-representativas locales (gobierno y partidos) en las experiencias de las ciudades de Córdoba, Gualeguaychú, Paysandú y Montevideo. En primera instancia, se presenta un modelo teórico de análisis con tipos ideales, que expresan formas posibles de relacionamiento. Seguidamente, utilizando el modelo planteado, se analizan los cuatro casos, en dos momentos diferentes, separados por una elección de gobierno local. Finalmente, se señala, en función de los casos estudiados, cómo en escenarios de participación plural y alcance restringido la mayor institucionalización generaría un estímulo para ir paulatinamente ampliando el alcance.

Palabras clave: presupuestos participativos, alcance, participación, institucionalización.

Abstract

The aim of the article is to analyze the forms of articulation between the participatory budget and the local political-representative institutions (government and parties) in the experiences of the cities of Córdoba, Gualeguaychú, Paysandú and Montevideo. A theoretical analysis model with ideal types expressing possible forms of articulation is presented in 
the first instance. Then, using the model presented, the four cases are analyzed in two moments of time separated by a local government election. Finally, it is pointed out how in scenarios of plural participation and restricted scope greater institutionalization could generate an incentive to gradually expand the scope.

Key Words: Participatory budgets, scope, participation, institutionalization.

\section{INTRODUCCIÓN}

El presupuesto participativo (PP) es actualmente la política participativa más difundida en el mundo. Siguiendo a Goldfrank (2006), puede definirse como un proceso participativo en el que los ciudadanos, de manera individual o representando a organizaciones cívicas, contribuyen en la toma de decisiones del presupuesto público. La forma como se da este proceso, el tipo de decisiones que se pueden tomar y su carácter resultan muy variados, dando lugar a multiplicidad de diseños aplicados a lo largo del mundo. Además del diseño institucional, existen variables vinculadas al contexto en el que se aplica la política, que llevan a diferentes tipos de articulaciones entre la participación ciudadana y las instituciones representativas.

En ese sentido, este artículo, basado en una investigación mayor, busca analizar los vínculos entre la participación en el PP y las tradicionales formas de representación político-partidaria, así como la manera en que esta complementa o se subordina a las instituciones de la democracia representativa. El estudio se realiza en cuatro ciudades: dos argentinas (Córdoba y Gualeguaychú) y dos uruguayas (Paysandú y Montevideo). Todas estas experiencias están atravesadas por elecciones de gobierno local durante el 2015, lo cual posibilitó analizar el fenómeno en la edición anterior a las elecciones y en la primera edición del nuevo periodo de gobierno. El periodo electoral se observó como un contexto idóneo para procesar trasformaciones en el diseño y funcionamiento de la política. Algunos cambios se observaron en el trabajo de campo realizado en la siguiente edición a las elecciones y otros, que tomarán más tiempo para ejecutarse, solo se analizan desde las modificaciones en el diseño institucional.

La pregunta de la investigación es: ¿bajo qué condiciones el PP funciona como un instrumento democrático de definición de los asuntos públicos?

A su vez, se plantean dos preguntas específicas: por una parte, ¿qué variables o dimensiones condicionan las estrategias del gobierno y los partidos políticos en relación con el PP? y, por otra parte, ¿cuáles son las 
trasformaciones que se generan en las experiencias en el contexto de elecciones locales?

Para analizar las formas de articulación se consideran dos variables centrales, a saber: el alcance de la política y el tipo de participación ciudadana. En base al comportamiento de estas variables se construyó un modelo teórico con escenarios típicos ideales con el que se analizaron las experiencias concretas. La variable alcance refiere a la capacidad efectiva de la herramienta de generar transformaciones en el territorio, lo cual en este trabajo se operacionalizó en: $a$ ) incidencia de las propuestas financiadas, $b$ ) coordinación entre el pp y demás áreas del gobierno local, y c) capacidad del pP para orientar otras políticas públicas.

Por su parte, la variable participación ciudadana refiere al tipo de vínculo que se genera entre el participante del pP y las instituciones de representación política (gobiernos y partidos, entre otros). La relación puede variar en un continuo entre autonomía y dependencia de la participación, en función de cuán libres o influenciadas son las decisiones que se toman en el pP respecto a la política. Hay dos aspectos que se consideran en la investigación: a) influencia de lógicas partidarias en la participación, y b) papel de ciudadanos y gobierno en la definición de prioridades en el PP.

Los hallazgos analizados fueron obtenidos a partir de entrevistas semiestructuradas realizadas a técnicos del PP, responsables políticos y ciudadanos (participantes y no participantes). Estas se realizaron en dos momentos: antes de las elecciones (en el 2015), sondeando el funcionamiento de la última edición del PP y las proyecciones de la política en el periodo electoral; y luego de las elecciones (durante el 2016), recabando información sobre la primera edición del nuevo gobierno. Se realizaron un total de 65 entrevistas. Asimismo, se efectuaron observaciones de asambleas durante la primera edición del nuevo periodo de gobierno en Gualeguaychú y Córdoba. Montevideo no prevé asambleas en su diseño y Paysandú interrumpió ese año la realización del ciclo, por lo que no correspondieron observaciones en estas localidades.' Se recurrió, a su vez, al análisis documental de normativas de los PP, organigramas de gobierno, prensa digital y plataformas electorales de candidatos a intendente.

En relación con la selección de los casos, el primer elemento que se consideró fue que las cuatro experiencias estuvieran atravesadas por procesos elec-

La primera edición de Pr, luego de las elecciones en Paysandú, a diferencia de las otras experiencias, fue a finales del mismo 2015, a pocos meses de asumir el nuevo gobierno. 
torales en los gobiernos locales, lo que permitió analizar las variaciones antes y después. Luego, se buscaron aspectos diferenciales en los diseños institucionales, la presencia de un escenario heterogéneo en elementos que se presupone podrían incidir en la articulación entre gobierno y participación. De esta manera, se consideraron las formas previstas para tomar decisiones, el papel de los delegados y la importancia de las asambleas en el proceso.

\section{Figura 1}

\section{Características consideradas para la selección de casos}

\begin{tabular}{|c|c|}
\hline $\begin{array}{l}\text { Córdoba } \\
\text { - Delegados toman decisiones sobre qué } \\
\text { financiar y qué no. } \\
\text { - Las asambleas son fundamentales en el } \\
\text { proceso. } \\
\text { - Toma de decisiones por consenso. }\end{array}$ & $\begin{array}{l}\quad \text { Gualeguaychú } \\
\text { - No existen delegados en el proceso. } \\
\text { - Las asambleas son fundamentales en el } \\
\text { proceso. } \\
\text { - Toma de decisiones por consenso (si no hay } \\
\text { acuerdo, en última instancia, se puede con- } \\
\text { vocar a votaciones). }\end{array}$ \\
\hline $\begin{array}{l}\quad \text { Paysandú } \\
\text { - No existen delegados en el proceso. } \\
\text { - Tiene reuniones, pero no toman defini- } \\
\text { ciones (son informativas). } \\
\text { - Se decide por votación (universal y se- } \\
\text { creta). }\end{array}$ & $\begin{array}{l}\text { Montevideo } \\
\text { - Existen delegados que integran comisiones } \\
\text { para evaluar las normas antes de empezar, } \\
\text { pero no definen las financiaciones. } \\
\text { - No hay asambleas. } \\
\text { - Se decide por votación (universal y secreta). }\end{array}$ \\
\hline
\end{tabular}

Fuente: elaboración propia

\section{ASPECTOS TEÓRICOS}

\subsection{El alcance de la política}

La incidencia de las propuestas es un elemento central para analizar el alcance de la política. La discusión de la literatura sobre este punto se centra en la pertinencia de que los PP aborden situaciones puntuales (cotidianas) o que, por el contrario, busquen articular propuestas de mayor escala, capaces de pensar en problemas más abarcativos en términos de incidencia (con una visión de desarrollo de la ciudad o la región). 
En general, los autores no son afines a los Pp circunscriptos a problemas estrictamente cotidianos y ligados al barrio, ya que dejan fuera de la discusión temáticas con mayor alcance social y político y contribuyen a que el pP se subordine a las instituciones de la democracia representativa (Montecinos, 2009, 2012; Cabannes, 2004). Sobre este punto, Cabannes (2004) propone avanzar en el debate, en relación con si el pp es un instrumento que ocupa un vacío de la representatividad, y por tanto un elemento central de la democracia representativa, o si es un espacio de democracia participativa de la proximidad, circunscripto a los problemas del barrio (Montecinos, 2009). Tanto Cabannes (2004) como Montecinos (2009) afirman que en los procesos en los que los ciudadanos se limitan a resolver cuestiones puntuales del vecindario, se produce una situación de subordinación del PP a las instituciones de la democracia representativa, las que cobrarían protagonismo en ese tránsito de lo barrial a la ciudad.

Otro aspecto destacado en materia de alcance es el establecimiento de canales de comunicación entre el PP y otras políticas públicas. Esto aumenta el potencial del pP de influir en la orientación de políticas, al tiempo que le permite desarrollarse, considerando un marco estratégico utilizado por otras instancias del gobierno y dotando de un determinado sentido de desarrollo local a sus inversiones (Montecinos, 2012; Welp, 2015).

\subsection{La participación en el PP y sus vínculos con el alcance}

Para el análisis de los casos de Diadema, Osasco, São Bernardo do Campo y Guarulhos, Romão (2014) propone una tipología de funciones donde resulta clave que las oficinas de planificación sean cercanas y la distribución de participantes en el Consejo (entre sociedad civil y gobierno) sea equitativa, pero que los consejeros sean cercanos al gobierno. Por otro lado, el PP tendrá un rol central en la estrategia de movilización de militantes y fuerzas políticas con afinidad hacia el Gobierno, cuando su coordinación esté más vinculada a la unidad de participación, no a la de planificación, y haya un mayor número de participantes de la sociedad civil en el Consejo. En estos casos, el pp tenderá a una función más cercana al diálogo con la ciudadanía que a la toma de decisiones. El tercer tipo planteado por el autor es el caso en el que el Gobierno implementa el PP, pero sin demasiadas convicciones, por lo que la herramienta se encuentra vacía de sus objetivos iniciales y se «desnaturaliza» (Welp, 2015). 
Welp (2015) entiende que este trabajo de Romão conduce a sugerir que el PP funciona mejor cuando se da una relación de mutuo beneficio entre los promotores (el Gobierno) y los ciudadanos más cercanos a sus políticas. En cambio, cuando se da la situación contraria, se desnaturaliza y se pierde el sentido. Ello implica que el PP es más poderoso cuanto más subordinado es el proyecto al partido de gobierno.

Desde otra perspectiva, Bacqué, Rey y Sintomer (2005) presentan tres escenarios posibles de articulación entre participación y representación.

En primer lugar, estarían los escenarios de removilización política a través de dinámicas participativas, en los que la participación ciudadana aparece como un complemento de proximidad de las democracias representativas. En este caso la participación puede actuar de dos maneras: como una instancia de consulta, donde se mantiene la definición en los representantes políticos, o como una instancia de definición, pero ligada a problemáticas domésticas y careciendo de incidencia en las grandes orientaciones de las políticas municipales.

El segundo escenario posible sería el de sustitución de un modo de participación por otro, en donde la participación se desarrolla en un campo en el que despliega su propia capacidad de decisión en dominios muy específicos. Aquí la participación se mueve sin necesidad de articularse con las instituciones representativas. Las ONG en los países subdesarrollados son mencionadas como ejemplos, ya que raras veces articulan con gobiernos locales y sus administraciones.

Finalmente, un tercer escenario es el de combinación entre formas convencionales y no convencionales de participación. En este caso, se apuesta por dotar la participación de un verdadero poder, buscando, de esta forma, dinamizar el sistema representativo. La participación disparada a través de diferentes dispositivos se convierte en una especie de cuarto poder.

Como sostienen Velázquez, González y Rodríguez (2008), los aportes de Tatagiba y Teixeira analizando el fenómeno en la ciudad de San Pablo presentan lo que se podría denominar un cuarto escenario posible, en donde las instancias de participación quedan contaminadas por las lógicas del juego político, propio del proceso de conformación de mayorías electorales. La participación se mueve aquí en función de las lógicas de los partidos políticos. 


\subsection{Institucionalización}

Si bien no existe una definición única de institución, se puede concebir como el conjunto de reglas del juego en las que tiene lugar la acción. Son:

[...] reglas usadas por los individuos para determinar quién y qué está incluido en las situaciones de decisión, cómo se estructura la información, qué acciones pueden adoptarse y en qué secuencia y cómo las acciones individuales serán agregadas en decisiones colectivas (Kiser y Ostrom, 1982: 179).

En igual sentido, Hall refiere a ellas como: «las reglas formales, los procedimientos de acuerdo y las prácticas estándar operantes que estructuran la relación entre individuos en las diversas unidades de comunidad política y económica» (Hall, 1986: 19).

Tanto la regularidad, entendida como la existencia de reglas preestablecidas que regulan las acciones, como la continuidad, comprendida como la permanencia en el tiempo de esas normas, son elementos clave de las instituciones (Scartascini y Tommasi, 2013: 1).

Si bien los estudios que incorporan la variable institucionalización en el estudio del pP lo hacen restringiéndola al análisis de la existencia de reglas formales, la propuesta que se presenta aquí es ampliar la mirada de la institucionalización hacia un plano que trascienda el aspecto jurídico, incorporando además elementos como la aceptación de los actores con capacidad de modificar reglas y el aval o aceptación de la ciudadanía. Estos, combinados con el elemento normativo, indican los niveles de institucionalización de la política. En otras palabras, la idea es una combinación de reglas del juego, que impiden que el pP sea un mero instrumento en manos del político de turno, y un fuerte aval ciudadano, que conduzca a considerar que no es posible eliminar el mecanismo.

Siguiendo esta línea, la hipótesis es que tanto el control político de la herramienta como su capacidad de sostenerse en el tiempo se ven afectadas por la mayor o menor institucionalización de la política. Esto implica que la mayor institucionalización en términos jurídicos podría atenuar el control político del Gobierno de turno, mientras el mayor aval ciudadano podría conducir a observar que no es posible eliminar el PP, al tiempo que podría estimular su desarrollo como herramienta democrática. 


\section{MODELO ANALÍTICO}

En la figura 1 puede verse la modelización de escenarios posibles surgidos de la combinación de las variables alcance y participación. Esto da lugar a cuatro grandes escenarios dentro de los cuales se establecen dos variantes, resultando un modelo de ocho escenarios ideales.

\section{Figura 2}

\section{Modelización analítica de escenarios de PP}

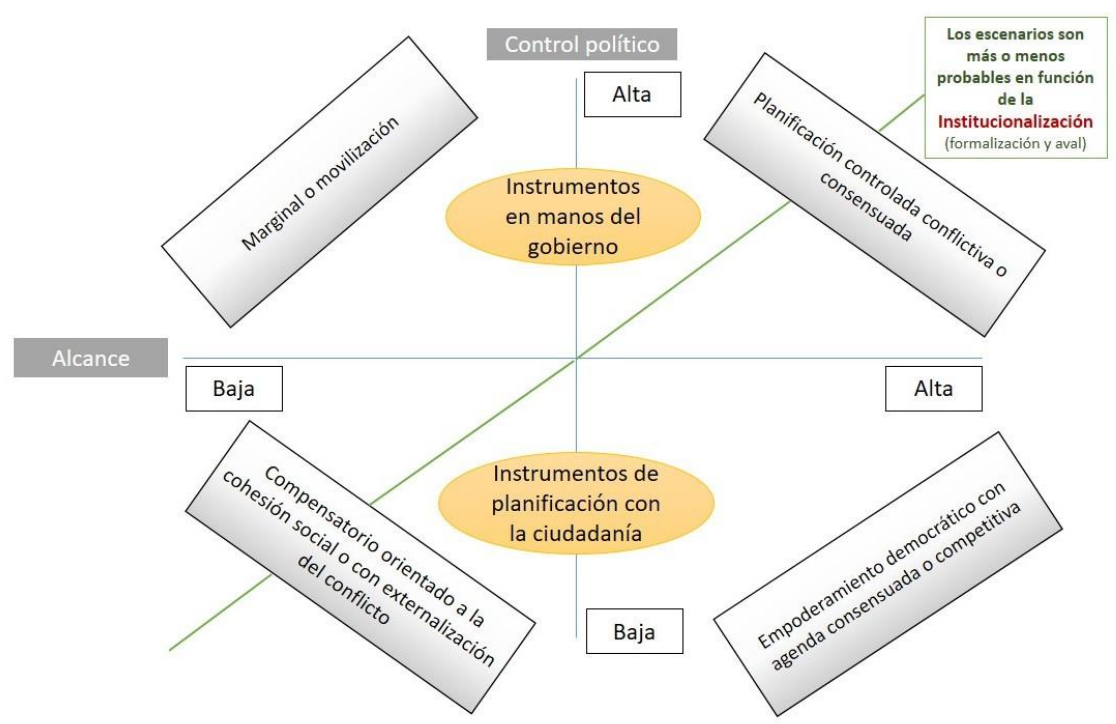

Fuente: elaboración propia

Se pueden definir de la siguiente forma:

1) Empoderamiento democrático: es el PP con mayor potencial para el desarrollo y exhibe una relación de complemento entre participación y representación. El alcance de la política es alto y la participación no está controlada políticamente. Esto implica una convocatoria abierta y una participación plural que toma definiciones de manera autónoma, sin verse influenciada por lógicas e intereses políticos. El mayor alcance se deriva de propuestas que generan efectos más allá del espacio micro de los grupos u organizaciones. Exhibe, a su vez, una orientación de desarrollo que considera y puede influir en los 
planes y políticas existentes en el territorio. O sea, el PP no se concibe como una política aislada y desconectada del resto de la gestión pública.

Dependiendo de las dinámicas participativas que se generen, encontramos dos variantes: de agenda consensuada o competitiva. a) Empoderamiento democrático con agenda consensuada: los ciudadanos, basados en diagnósticos compartidos, realizan una priorización y generan acuerdos, cuyos alcances trascienden el espacio micro de los grupos y organizaciones, logrando efectos sobre porciones más amplias de la comunidad. El PP original de Porto Alegre sería un ejemplo cercano a este modelo ideal. b) Empoderamiento democrático con agenda competitiva: también presenta un alto efecto en las propuestas y bajo control político de la participación, pero las inversiones revisten más dispersión que la agenda consensuada debido a que las propuestas aprobadas se derivan de una competencia (generalmente mediante el voto universal y secreto) entre una amplia variedad, que surgen en los diferentes grupos que participan. Pese a la dispersión de propuestas y a la competencia, en este modelo las inversiones realizadas deben asegurar un efecto que trascienda el espacio de los grupos que la promueven, aumentando así el alcance de las trasformaciones.

2) Modelos compensatorios: cuando la herramienta queda fuera del control político y su alcance es bajo, puede observarse un escenario en el que el PP tiene una función compensatoria, con dos variantes: orientado a la cohesión social o a la externalización del conflicto. a) Compensatorio orientado a la cohesión social: busca minimizar las carencias en las condiciones de vida de los participantes. Los grupos y organizaciones obtienen pequeñas contribuciones orientadas a resolver problemas concretos. Pese al foco en problemáticas domésticas y aunque se carece de incidencia en las grandes orientaciones de las políticas, el PP cumple una función de cohesión social involucrando a grupos formales e informales que generan consensos sobre cómo distribuir los recursos escasos. No se produce una competencia, sino que, a través de la deliberación, se buscan acuerdos $y$ se fortalecen los vínculos sociales. b) Compensatorio con externalización del conflicto: al igual que el anterior, posee un carácter compensatorio, con un bajo control político y bajo alcance, pero no se cumple la función de cohesión social, sino que se plantea una disputa por los recursos. Se trata de un PP donde los problemas cotidianos a ser financiados, en un contexto de escasos recursos, deben demostrar primero su superioridad en términos de apoyos ciudadanos. Son PP diseñados para la competencia, más que para el consenso, y su principal desafío en términos democráticos son las asimetrías sociales existentes. 
3) Planificación controlada: en este modelo el Gobierno o partido de gobierno toman las decisiones más importantes. La definición de ciertas temáticas y su abordaje expresan un interés del municipio más que de la ciudadanía. Como el Gobierno dirige el proceso y direcciona la inversión hacia áreas estratégicas, los montos son elevados y las propuestas aprobadas tienen una alta capacidad de impacto. En síntesis, se trata de escenarios en donde el PP exhibe un alcance elevado, pero un rol muy pobre de la ciudadanía. También es posible admitir dos variantes: consensuada o conflictiva, en base a si existe competencia o acuerdos entre ciudadanos para la obtención de recursos, aunque en un escenario en que la mayoría de las definiciones tienen una orientación que escapa a su voluntad.

4) Cuando el alcance es limitado y el instrumento queda bajo el control político, observamos dos posibles escenarios. a) Marginal: el Gobierno lleva adelante el pp con el único fin de mostrarse como un gobierno moderno. El prestigio de la política y el aval de organismos nacionales e internacionales llevan a que un gobierno local vea en la herramienta una oportunidad para mostrarse como un gobierno avanzado en términos democráticos. El resultado es un PP controlado políticamente y con alcance limitado. $b$ ) Movilización: el Gobierno utiliza el PP para movilizar militantes y fuerzas políticas con afines. No se destinan grandes montos, ni se tratan temas importantes de la agenda departamental, pero a través de él se mantiene activa la red de militantes y se reclutan nuevos, siendo el PP un instrumento netamente político.

\section{PRESENTACIÓN DE LOS CASOS}

\subsection{Córdoba}

La ciudad de Córdoba es la capital de la provincia homónima en Argentina, cuenta con 1329604 habitantes y con una gran dispersión territorial. El proceso de descentralización de la ciudad creó centros de participación vecinal (CPC) y las políticas y los ámbitos de participación ciudadana estuvieron anclados sobre ellos mediante Juntas de Participación Vecinal (JPV), siendo el PP uno de los principales instrumentos de deliberación y toma de decisión ciudadana.

La normativa establece que en el pp hay dos ámbitos territoriales de participación, los CPC (actualmente hay once) y, dentro de ellos, las zonas barriales. Se prevén varias ruedas de asambleas participativas, con una participación 
abierta, orientada al diagnóstico y la priorización de problemáticas. Luego, en el propio proceso, eligen representantes para la fase final denominada taller de PP. Como se verá en el análisis, en los hechos esto funciona muy distinto.

\subsection{Gualeguaychú}

La ciudad de Gualeguaychú es la capital del departamento homónimo, ubicado en la provincia de Entre Ríos (Argentina). El municipio comprende la ciudad y una área rural, sumando una población de 83116 habitantes. Aquí el PP no está inserto en un proceso de descentralización de la ciudad, como sucede en Córdoba y Montevideo, pero forma parte de una estrategia gubernamental para canalizar la participación ciudadana. Existen cuatro formas de PP: el general, el joven, el pP de adulto mayor y el de turismo. El presente análisis se realiza sobre el pp general.

La metodología consiste en una convocatoria anual para presentar propuestas de carácter individual o mediante organizaciones. Pueden participar los vecinos mayores de 16 años que estén habilitados para votar. El proceso implica cuatro reuniones asamblearias, organizadas en nueve zonas, donde se presentan y discuten las propuestas, vinculadas a obras o servicios comunitarios.

No existe instancia en la que los ciudadanos sean representados por delegados, se mantiene siempre una participación directa. En la reunión final, se busca el consenso entre los presentes sobre qué proyectos financiar, considerando los montos máximos por distrito. Las propuestas no tienen un monto máximo dispuesto, de manera que puede interpretarse que el máximo corresponde al monto del distrito.

\subsection{Paysandú}

La ciudad de Paysandú es la capital del departamento del mismo nombre y posee una población de 74575 habitantes. El PP se originó con la llegada del Frente Amplio (de orientación progresista) por primera vez al gobierno de la ciudad, en el 2005 .

Desde la creación del pP hasta la fecha se efectuaron muchos cambios en el diseño. Algunos, producto del cambio de orientación política del Gobierno, y otros, dentro del mismo periodo de gobierno. Sin embargo, hay una estructura básica que se mantuvo en todas las ediciones que conlleva una presentación de propuestas por distrito (con montos máximos predefinidos) y una posterior 
elección mediante del voto universal y secreto, generando un ordenamiento de proyectos financiables, en orden descendente, hasta donde alcancen los montos. La convocatoria es abierta, los grupos y organizaciones presentan sus propuestas y estas deberán competir con otras en una elección donde se convoca a la ciudadanía a participar. Las asambleas, si bien son obligatorias, tienen un componente básicamente informativo, no se realizan diagnósticos, ni se toman decisiones sobre la financiación.

\subsection{Montevideo}

Montevideo es la capital de Uruguay con una población de 1319108 habitantes. Con la llegada del Frente Amplio a la intendencia en 1990 junto a Tabaré Vázquez, el Gobierno de la ciudad inició un proceso de descentralización que sería el marco general dentro del cual se inserta el PP. La ciudad está dividida en ocho municipios, los que tienen divisiones administrativas en centros comunales zonales y cada uno de ellos cuenta con un concejo vecinal (CV). Estos son órganos consultivos y deliberantes de participación ciudadana integrados por vecinos de la zona, electos directamente por la ciudadanía.

El pP involucra una convocatoria bianual a presentación de proyectos. Dicha presentación puede efectuarse individualmente o mediante organizaciones, no presentándose instancias de asambleas. La base territorial son los ocho municipios de la ciudad de Montevideo, existiendo una división de recursos en partes iguales entre las zonas. Las temáticas deben enmarcarse en alguna de estas áreas: 1) Infraestructura y servicios; 2) Desarrollo y promoción comunitaria y social; 3) Género y diversidad, incluyéndose aquí a: a) Juventud, infancia y adolescencia; $b$ ) Adultas/os mayores; c) Equidad racial y migrantes; d) Discapacidad; e) Cultura y deportes; y otros. Las propuestas deben estar destinadas a concretar intervenciones de interés vecinal asegurando el uso público de las realizaciones.

Un aspecto interesante de su diseño es su modalidad de autorreglamento, que implica que antes de iniciar una edición se evalúe el funcionamiento de la anterior y se propongan ajustes a las normas. Esto lo hace la Comisión Departamental de Presupuesto Participativo (CDPP), integrada por representantes de los $18 \mathrm{CV}$ y un representante de la intendencia. 


\section{COMPARACIÓN DE LOS CASOS EN FUNCIÓN DEL MODELO}

En la figura 2 puede apreciarse una ubicación estimada para cada uno de los cuatro casos en la primera edición (previa a las elecciones locales). En los extremos del diagrama se colocó el tipo ideal, mientras que los casos concretos se ubican más o menos cerca de este tipo en función de las características que comparten con él en su funcionamiento real. Se debe considerar que la representación es una herramienta ilustrativa que ayuda a visualizar diferencias y movimientos en las experiencias, pero no se basa en cálculos que marquen una posición precisa.

Figura 3

Ubicación de los PP en el modelo (antes de las elecciones)

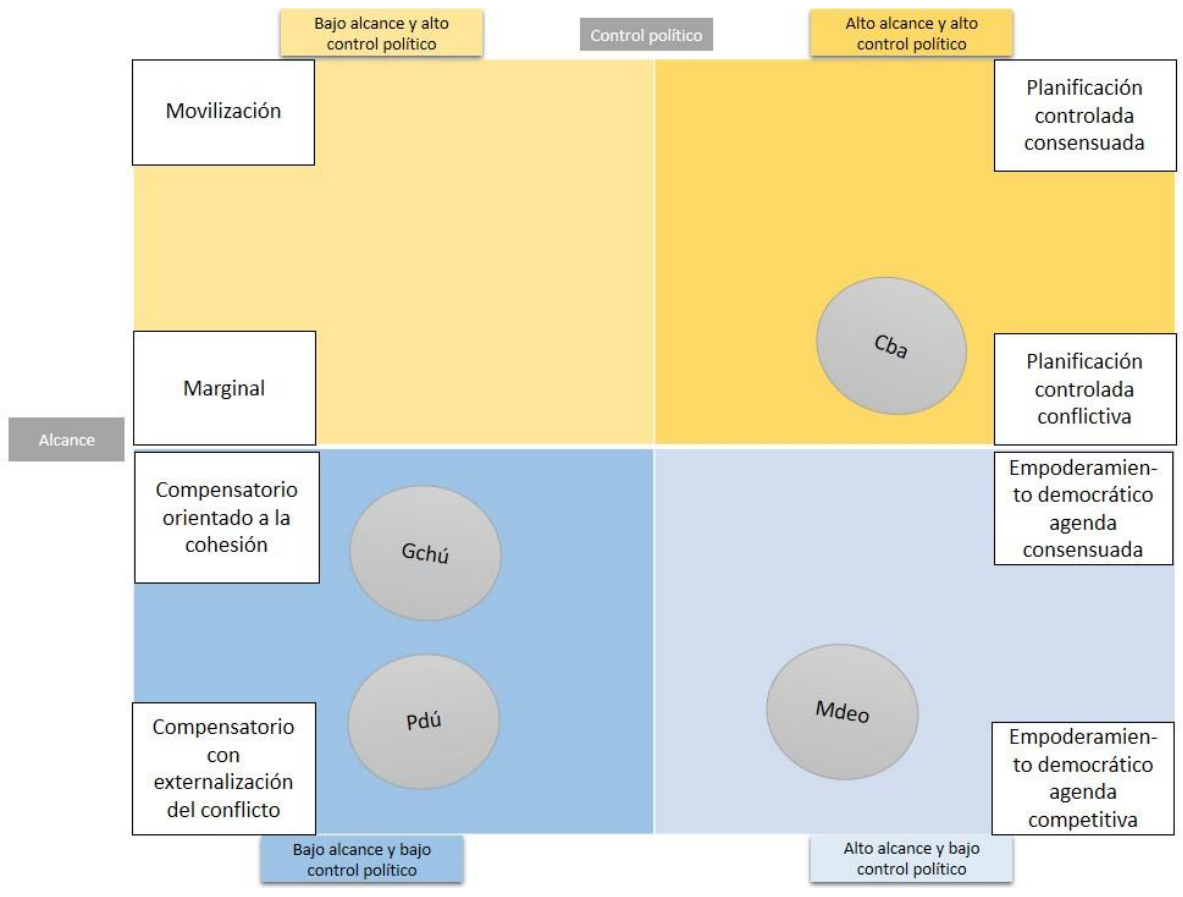

Fuente: elaboración propia

Los casos de Gualeguaychú y Paysandú se acercan a los modelos compensatorios. En ambos, las propuestas financiadas refieren a pequeñas transformaciones que benefician a grupos concretos $u$ organizaciones, careciendo de efectos que involucren a la comunidad en su conjunto o sectores 
más amplios. Por otro lado, las propuestas no exhiben conexiones entre sí y no parten de un diagnóstico zonal, por lo cual, se produce una dispersión de las inversiones sin un propósito común.

La orientación hacia asuntos de baja escala le ha dado a la herramienta, en ambos casos, una utilidad concreta para los vecinos que va más allá de los aspectos partidarios, ya que convoca a participar a una pluralidad de actores con afinidades políticas diferentes que actúan motivados por los intereses concretos de la organización más que como militantes.

En Gualeguaychú se aproxima al modelo compensatorio orientado a la cohesión social, que busca evitar la competencia entre propuestas y queda relegada como última opción, siendo además desalentada por los promotores del Gobierno. Existe un acuerdo inicial de los participantes de evitar que haya propuestas que no obtengan financiación, aunque ello implique que las ideas originales de los grupos deban reducirse considerablemente, y son muy pocas las propuestas que se descartan en la asamblea final. Más allá de algunos conflictos puntuales, en donde se reclama priorizar proyectos que tienen como población objetivo a sectores vulnerables, se acepta esta lógica de compartir los recursos escasos. Este elemento le da al PP de Gualeguaychú un efecto potencial más allá de las pequeñas inversiones que realiza a escala de la vida cotidiana: la contribución al fortalecimiento de la cohesión social.

En Paysandú, en cambio, el bajo control político y el bajo alcance de las propuestas se dan en un escenario de disputa por los escasos recursos. Por ello se puede decir que el PP no apunta a generar lazos solidarios entre las organizaciones y grupos del territorio. Si bien existe la posibilidad de generar alianzas entre dos organizaciones del mismo rubro y repartir el dinero, lo que predomina es la competencia y existen ciertas instituciones que ya lograron una cierta base de apoyo territorial que las hace potenciales ganadoras. Se presentan, asimismo, instituciones que dependen del dinero del PP para su funcionamiento.

En el caso de Córdoba, el PP se acerca al modelo de planificación controlada consensuada. La idea original planteada en la ordenanza y el reglamento implica varias ruedas deliberativas donde se hacen diagnósticos a distinta escala, se priorizan problemáticas y se elaboran propuestas, en un escenario de participación abierta, en cuya instancia final se actúa a través de representantes electos en asamblea previa. Sin embargo, lo que se observó es que el proceso se simplifica a un solo taller en el que se presenta a los 
participantes (líderes sociales) ${ }^{2}$ un conjunto de propuestas por las que van optando en función de lo que consideren necesario para la zona.

No existe la posibilidad en los pP de Paysandú, Gualeguaychú y Montevideo de plantear ideas que emerjan de la ciudadanía; se trata de una dinámica en la que se pone a disposición de los participantes una gama de ofertas predefinidas por el Gobierno en función de sus posibilidades de gestión o de otros intereses. Esto lleva a que las principales definiciones estén en manos del Gobierno y no de la ciudadanía. Por ejemplo, existe una serie de talleres que dependen de la oferta anual de la Secretaria de Cultura de la municipalidad y, dentro de esa oferta, los participantes deben optar, no pueden elegir otro tipo de talleres o directamente decidir que en vez de talleres quieren gastar el dinero en otra propuesta. Se trata entonces de un PP con una participación poco plural, controlado desde el Gobierno en sus definiciones principales, donde no se producen conflictos o competencias para la obtención de recursos entre vecinos, y con un alcance de las propuestas de nivel medio, superando el porte de las obras de Gualeguaychú y Paysandú.

Por su parte, el pp de Montevideo se acerca al modelo de empoderamiento democrático con agenda competitiva. Tiene un impacto medio-alto de las propuestas financiadas y un bajo control político de la participación. La percepción es que la participación se produce motivada por fines utilitarios, buscando beneficios para las organizaciones participantes o para el vecindario, y no por móviles político-partidarios. El hecho de no tener un espacio de deliberación y decidir las preferencias a través del voto universal y secreto le da a la herramienta una cierta independencia de la voluntad política, pero, a su vez, como contracara, limita las posibilidades de pensar en términos de zonas y ver cuáles son los principales problemas y desafíos y las formas más creativas de hacerles frente. El resultado de la falta de diálogo y el carácter competitivo de la toma de decisiones da a las inversiones un carácter disperso e inconexo. No obstante, la herramienta ha mostrado un potencial importante para llevar adelante una variedad de propuestas que emergen de la ciudadanía, colocándole muy pocas restricciones a la hora de formular sus proyectos.

Uno de los aspectos que se volvió obligatorio a la hora de formular las propuestas es que estas posean un efecto más allá de las organizaciones que las proponen. Este componente, en el que se ha trabajado fuertemente en la

\footnotetext{
Contrario a lo que dice la norma, el Gobierno convoca únicamente a integrantes de las JPV (compuesta por
} representantes de los centros vecinales y algunas organizaciones de la sociedad civil). 
edición del 2016, potencia la capacidad de alcance de las propuestas y genera mayores efectos sobre la comunidad.

Luego de las elecciones se realizó un análisis del diseño y del funcionamiento de la siguiente edición del PP en las cuatro localidades. La siguiente imagen representa la cercanía o distancia en relación con los modelos bajo esta nueva realidad. En algunos casos, donde los cambios más significativos aparecieron luego de la edición del 2015-2016, se definió incorporar estos cambios analizados únicamente desde el diseño.

\section{Figura 4}

Ubicación de los PP en el modelo (después de las elecciones)

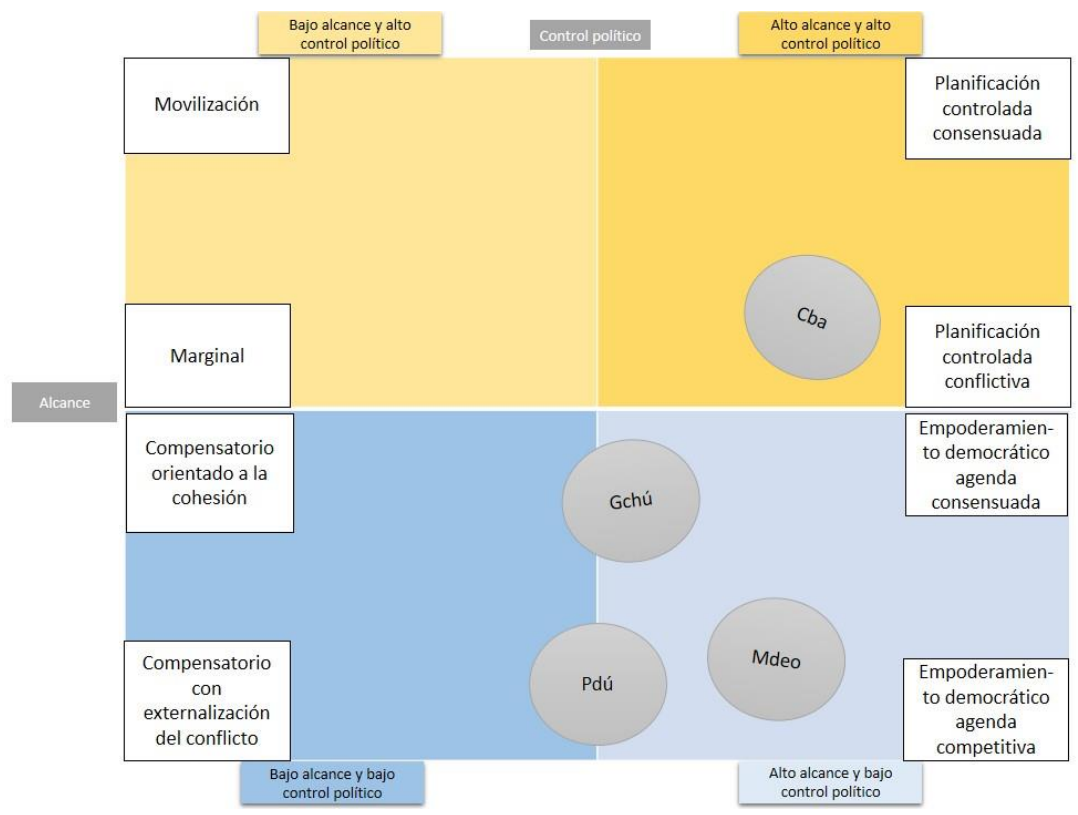

Fuente: elaboración propia

Los casos que experimentaron más cambios fueron los PP de Gualeguaychú y Paysandú. En ambos, las modificaciones no se pusieron en marcha en la edición observada, sino que ocurrieron en el 2017 en Paysandú y en el 2018 en Gualeguaychú. Esto se debió al procesamiento y la discusión interna que generaron las modificaciones. En el caso de Paysandú, no se tenía claro desde el Gobierno qué modificaciones podían darle un nuevo empuje a 
la política, por lo cual decidieron parar la ejecución en el 2016 y evaluar la política antes de aplicar cambios. En Gualeguaychú, además de diseñar los cambios, se debía modificar la ordenanza, de manera que durante varias ediciones el PP continuó con el mismo diseño que se aplicó en el anterior periodo de gobierno. En ambas, las modificaciones realizadas le colocaron rasgos de los modelos de empoderamiento democrático a la política.

En Gualeguaychú, el Pp se aleja un poco del modelo compensatorio orientado a la cohesión social, aunque mantiene algunos rasgos y se acerca en algunos aspectos al modelo de empoderamiento democrático con agenda consensuada. En la nueva propuesta se combina la lógica de las pequeñas trasformaciones (dispersas y orientadas a actores particulares) con proyectos comunitarios que trascienden los intereses particulares y que se focalizan en los principales problemas que tiene la zona. Esto obliga a pensar primero en los problemas y el diagnóstico, priorizarlos y ver cuál de ellos se prefiere abordar en esta edición y luego definir de qué manera se contribuirá a mejorar esa situación. En términos de diseño, el Pp de Gualeguaychú se vuelve mucho más potente en relación con el alcance, asegurando una propuesta de porte medio ( $25 \%$ del total de presupuesto destinado al distrito), pensada con lógica de zona y no de grupo. Se deberá analizar el funcionamiento efectivo de la dinámica para evaluar si en los hechos realmente logra el cometido.

Por otro lado, al no eliminar la lógica de microproyectos particulares, el PP no pierde del todo el componente compensatorio, lo cual le sigue dando un atractivo para la pluralidad de grupos que participan movidos por sus propios intereses (sin vínculos políticos), pero que ahora también deberán pensar en los problemas de la zona. Por su parte, el presupuesto destinado a la herramienta no permite pensar en un abordaje integral de los problemas más complejos que se puedan diagnosticar en las diferentes zonas. Este aspecto también lo distancia del modelo ideal de empoderamiento democrático.

En Paysandú también se apreciaron, a partir de la edición del 2017, algunos componentes del diseño propios del modelo de empoderamiento democrático, aunque en este caso con agenda competitiva y no consensuada. El PP mostró una estructura de pocos proyectos y de mayor porte, en especial los relacionados con infraestructura y obras. Los cambios efectuados implicaron que los ejes temáticos se redujeron de cinco a dos: 1) obra e infraestructura y 2) desarrollo comunitario y social. Con ello también disminuyó la cantidad de propuestas que se podían aprobar por zona, pasando de once proyectos en el 2015 a tres en el nuevo diseño. Asimismo, en cada zona solo se puede aprobar un proyecto del rubro obras e infraestructura por un 
total de 50 ooo dólares; esto podría habilitar la realización de obras de porte medio y efecto zonal, aunque ello no está garantizado, ya que también se admite en el rubro la presentación de propuestas de reparaciones edilicias de instituciones. Esto podría llevar a que se siga primando una lógica de competencia entre propuestas emergentes, que benefician a las propias organizaciones más que a la comunidad en su conjunto.

Otro aspecto que potencia el alcance del Pp de Paysandú tiene que ver con el aumento del presupuesto destinado a la política, que junto con la menor dispersión de las propuestas aprobadas les da un efecto potencial mayor. Entonces, a partir del 2017, Paysandú muestra un diseño que favorece obras de mayor porte, pero que se impulsan desde la lógica de intereses particulares de los colectivos que participan. En ese sentido, no logra, como lo hace Gualeguaychú, pensar desde una lógica comunitaria donde los participantes no pueden solicitar, en ese subespacio, beneficios para su grupo. A este respecto, para continuar en dirección hacia un modelo de empoderamiento ciudadano, el PP de Paysandú debería garantizar un mayor efecto comunitario de las propuestas y eso podría lograrse con espacios específicos, como lo hizo Gualeguaychú, o con contrapartidas para el aprovechamiento comunitario, como lo hizo Montevideo.

Por su parte, los cambios en el diseño del 2017 de Paysandú incluyen una novedad en relación con quién puede presentar propuestas. Por primera vez, desde su creación, se pone límite a las instituciones que pueden presentar propuestas, dejando fuera a aquellas que dependen presupuestalmente del Gobierno central: escuelas, policlínicas, liceos, etc. Esto aparejará cambios en el perfil de los participantes que se deberán analizar en un futuro.

En Córdoba no se advierten cambios, se mantiene el modelo de planificación controlada, con un perfil de participantes líderes sociales y un Gobierno que define con discrecionalidad qué cosas son las que se les ofrece a los participantes y cuáles no. Estos cumplen su rol de intermediarios entre el Gobierno y los vecinos, pero no logran una incidencia real en la definición del gasto. De los cuatro casos estudiados este es el que contribuye menos al empoderamiento democrático.

Finalmente, en Montevideo, el avance más interesante, que lo acerca aún más al modelo de empoderamiento ciudadano con agenda competitiva, es el de generar especificaciones en la normativa ya existente para regular el tema de las contrapartidas a las instituciones que presentan proyectos en su propio beneficio. Si bien estaba presente en ediciones anteriores la idea del impacto comunitario de las propuestas, en la edición del 2016 se hicieron avances 
importantes para darle concreción, mencionando con claridad las formas como deben darse estas contrapartidas, incorporando estos elementos al estudio de viabilidad y estableciendo las consecuencias del no cumplimiento de las mismas en la ejecución de la propuesta. De esta forma, pese a la orientación competitiva y a la ausencia de espacios deliberativos, el pP de Montevideo logró trascender el espacio micro de las instituciones y los grupos, obligando a levantar la mirada hacia la comunidad o un conjunto de instituciones dentro de ella. Por lo que se observó en algunos casos de proyectos ganadores, estas contrapartidas llevan a generar redes de trabajo interinstitucionales en torno a determinadas propuestas, fortaleciendo la cohesión social.

\section{INSTITUCIONALIZACIÓN: DIFERENTES NIVELES Y RELACIÓN CON EL ALCANCE, LA AUTONOMÍA Y LA PERMANENCIA}

Como se dijo, el concepto de institucionalización se compone de dos elementos: uno jurídico y uno sociológico. Por un lado, se observa el grado en el que el proceso se encuentra respaldado y regulado por normas jurídicas: constitución, leyes, ordenanzas, decretos, reglamentos (que se estudia aquí en diseño institucional); a mayor cantidad y jerarquía de las normas que regulan el proceso, mayor nivel de formalización o institucionalización en el sentido jurídico. Por otro lado, se incorpora la idea del respaldo ciudadano a la existencia y el funcionamiento de la política, o sea el componente de las normas sociales (que en parte deriva de participación). Un mayor convencimiento de la ciudadanía de los aportes de la herramienta a la calidad de la democracia indica una mayor institucionalización en este sentido.

En términos de formalización, se puede decir que ninguna de las experiencias obliga a los gobiernos locales a aplicar el PP mediante la Constitución o la ley. Sin embargo, las dos experiencias argentinas tienen el PP regulado a través de ordenanzas y reglamentos, lo cual significa que el ejecutivo local está obligado a aplicarlo salvo que se derogue la ordenanza, lo que requiere la intervención del órgano legislativo local. En cambio, las experiencias uruguayas se mantienen en un mayor nivel de informalidad, en donde no hay ni marcos nacionales que obliguen a los gobiernos departamentales a su aplicación (que tampoco existe en los casos argentinos), ni regulaciones por parte del órgano legislativo departamental, existiendo solamente un reglamento interno que emana del propio ejecutivo 
departamental. Lo que se observó es que en los casos estudiados estos niveles de formalización no afectan en gran medida la continuidad de la política; salvo situaciones puntuales, no hubo propuestas electorales que mencionaran explícitamente su intención de no continuarlo y los candidatos con posibilidades de ganar en todos los casos se refirieron a la continuidad del PP. Esto podría obedecer a que la política se ha instalado y tiene sectores de la sociedad que la valoran y defienden como herramienta, al tiempo que los gobiernos (y los que aspiran a llegar serlo) sienten que es necesaria para exhibir una gestión moderna que siga las orientaciones participativas de organismos nacionales e internacionales.

Por otro lado, se observó que los casos de Paysandú y Montevideo, de baja formalización, siguieron fielmente el reglamento, generando un proceso de participación que sigue las diferentes etapas que están pautadas en la normativa. En cambio, en el caso de Córdoba, que está regulado por ordenanza legislativa, el proceso participativo sufrió serias modificaciones por la vía de los hechos: se acortaron las etapas, se restringieron los temas, se cambió la modalidad de participación y se limitaron las personas convocadas. Nada de esto se realizó de acuerdo con la norma, lo cual evidencia que no siempre una mayor formalización implica mayor estabilidad y garantía de cumplimiento de los procesos.

El pp de Córdoba no sufrió cambios de una edición a otra (2015-2016), ni se avizoran cambios importantes. Tampoco cambió el Gobierno y tanto el intendente como el director del Pp continúan en su cargo. La campaña no mostró partidos que pusieran en tela de juicio la continuidad del PP, pero tampoco demasiados cuestionamientos ni grandes propuestas transformadoras. De hecho, el partido de gobierno solo prometió ampliar su alcance mediante una mayor inversión.

En los casos de Paysandú y Gualeguaychú, los discursos recogidos a través de entrevistas mostraron una política legitimada socialmente. Ese aval ciudadano actuó como un estímulo para darle continuidad y ampliar su potencial. Paysandú, dentro de las experiencias estudiadas, es la que más alternancia de partidos tuvo en el gobierno, se inicia con el Frente Amplio, luego el Partido Nacional y, en el 2015, nuevamente, retorna el Frente Amplio. En estas alternancias, resulta interesante ver cómo el Partido Nacional ingresa en el Gobierno en el 2010 poniendo en tela de juicio la continuidad del PP, dada su vinculación con la gestión frenteamplista; pero luego, motivado por la capacidad de la herramienta para llegar a diversidad de actores y el aval de la ciudadanía, decide mantenerlo, aunque con modificaciones y quitándole 
centralidad dentro de la oferta estatal 3 (Etchemendi, 2015). Tras la elección del 2015, ninguno de los candidatos puso en juicio su continuidad y se marcó además la necesidad de potenciar la herramienta. Se pudieron evidenciar algunos matices en los discursos del Partido Nacional y del Frente Amplio, que se orientaban a marcar diferencias en relación al futuro diseño de la herramienta.

En Gualeguaychú se observó una política que cuenta con amplia legitimidad social y un fuerte compromiso de los responsables de la Secretaría que lo ejecuta. ${ }^{4}$ Esto puede apreciarse como un elemento más de la institucionalización/consolidación que ha alcanzado la política en el lugar. Como correlato de esta alta institucionalización, los candidatos incluyeron propuestas importantes en relación con el futuro de la herramienta. De hecho, para uno de ellos, la propuesta del pp Social fue uno de los puntos centrales de su campaña electoral. Tras la victoria de Esteban Piaggio, 5 el PP, como se propuso en la campaña, cambió su posición en el organigrama y las políticas participativas pasaron de una dirección a una secretaría; Daniel Vaena, que estaba al frente de la dirección, pasó a ser secretario del nuevo órgano. En el 2007, tras modificar la ordenanza, el PP continuó sus transformaciones; modificándose el diseño institucional, buscando combinar las propuestas micro, con propuestas de mayor efecto comunitario.

El PP de Montevideo es una política muy instalada y es una de las pioneras en el mundo. Sin embargo, ha ido perdiendo centralidad en la oferta del gobierno, al punto de no aparecer mencionada explícitamente en el programa del Frente Amplio, aun cuando sí existe un eje referido a democracia participativa. Esta falta de jerarquización por parte del propio partido de gobierno debe ser interpretada en el escenario del año 2015, en donde el PP mostraba un descenso paulatino en la cantidad de participantes en su votación y donde, de acuerdo a encuestas, la herramienta no gozaba de altos niveles de popularidad.

Se presenta, entonces, un aval ciudadano débil, una clase gobernante que no se muestra entusiasmada con la herramienta y un nivel de formalización bajo. Sin embargo, pese a su perfil competitivo, el Pp avanzó en base a la

\footnotetext{
El 22 de octubre de 2010 se discute en la Junta Departamental (por iniciativa del partido de gobierno) el destino del pp y una de las opciones es la eliminación de dicha política.

4 El secretario asistía a todas las reuniones y, pese a la presencia de directores y funcionarios del pp, él se encargó de la moderación en los encuentros observados.

5 Es el candidato del Frente para la Victoria que representa la continuidad de la gestión de su antecesor Juan José Bahilo.
} 
experiencia y la autoevaluación que realizaron los funcionarios y los ciudadanos integrantes de los CV. Montevideo tiene el reglamento más completo de las experiencias estudiadas, donde se detallan de forma específica los procedimientos de cada etapa. Esto es el fruto de la actualización que se realiza por parte de esta CDPP. Las entrevistas muestran un buen clima de colaboración de las diferentes áreas de gobierno con el Pp, el que además se encuentra en una posición en el organigrama que está por fuera de los diferentes sectores lo que facilita pensarlo como una herramienta transversal. En la edición del 2016, se observaron avances que buscaron potenciar las propuestas con efectos comunitarios. Entonces, en Montevideo no se ve con claridad, como en Paysandú y Gualeguaychú, la relación entre el aval ciudadano y la emergencia de propuestas políticas para potenciar la herramienta; ello puede verse influido por el hecho de que su evolución parece haber estado más ligada a las definiciones de la CDPP que a las propuestas electorales y planificaciones del partido de gobierno.

\section{CONCLUSIONES}

De acuerdo con el modelo propuesto, el vínculo entre autonomía y participación es el que define el tipo de herramienta. En principio, es más probable la vinculación que propone Romão (2014) en la cual, a mayor alcance, menor es la autonomía y viceversa. Esto, desde el punto de vista teórico, lleva a pensar en que no es posible encontrar herramientas de empoderamiento democrático $\mathrm{y}$, de hecho, el desencanto que muchos tienen actualmente con la herramienta se relaciona con ello. Donde hay mucha autonomía, hay poco en juego, y en donde hay mucho en juego, la participación es controlada. En los casos estudiados se vio que la relación no es lineal y que es posible pensar en herramientas diseñadas originalmente para intervenciones de bajo impacto que desarrollan modificaciones metodológicas que propician que los participantes levanten la mirada del grupo a la comunidad y vayan ampliando el porte de las inversiones en un contexto de pluralidad participativa. Un aspecto que lleva a observar diferencias es que los diseños de los pp brasileños, para los que propone la relación Romão (2014), funcionan con Consejos de PP (integrados por ciudadanos y Gobierno), lo que genera implicancias diferentes en la relación entre participación y representación.

En los contextos electorales las políticas entran en debate y, en aquellos casos en los que el PP tiene una base amplia y plural de participantes, hay ma- 
yores estímulos para darle continuidad a la política y para ir ampliando su potencial.

\section{BIBLIOGRAFÍA}

Bacqué, Merie, Rey, Henri y Sintomer, Yves (2005). La démocratie participative, un nouveau paradigme de l'action publique? En Bacqué, Marie-Hélène, Rey, Henry y Sintomer, Ives (Dir.). Gestion de proximité et démocratie participative. Une perspective comparative (9-48). Paris: La Découverte.

Cabannes, Yves (2004). Presupuesto Participativo y finanzas locales. Programa de Gestión Urbana. Coordinación para América Latina y El Caribe. Ecuador: BID.

Etchemendi, Ernesto (2015). De Pintos (2005-2010) a Bentos (2010-2015), dos modelos de presupuesto participativo en Paysandú ciudad. Montevideo. Tesis de grado: UdelaR.

Goldfrank, Benjamin (2006). Los procesos de «presupuesto participativo» en América Latina: éxito, fracaso y cambio. Revista de Ciencia Política, 26(2), 3-28.

Hall, Peter (1986). Governing the Economy: The politics of state intervention in Britain and France. New York: Oxford University Press.

Kiser, Larry y Ostror, Elison (1982). The three worlds of action: a metatheoretical synthesis of institutional approaches. En Elinor Ostrom (Ed.). Strategies of Political Inquiry. California: Sage.

Montecinos, Egon (2009). El Presupuesto Participativo en América Latina. ¿Complemento o subordinación a la democracia representativa? Revista CLAD Reforma y Democracia, 44, 145-174.

Montecinos, Egon (2012). Diseños institucionales y participación ciudadana en presupuestos participativos: los casos de Chile, Argentina, Perú, República Dominicana y Uruguay. Comunicación presentada en XVII Congreso Internacional del CLAD sobre la Reforma del Estado y de la Administración Pública, Cartagena, Colombia.

Romão, Wagner (2014). For what? The political meaning of participatory budgeting. Chicago: LASA.

Scartascini, Carlos y Tommasi, Mariano (2013). Institucionalización de las instituciones políticas y su impacto sobre las políticas públicas. En Documento de trabajo del BID \# IDB-WP-287. 
Velásquez, Fabio, González, Esperanza y Rodríguez, Clara (2008). Participación ciudadana y representación política en contextos de conflicto armado. Bogotá: Corporación Región.

Welp, Yanina (2015). Participación ciudadana, poder y democracia: apuntes para un debate. Revista Argentina de Democracia Participativa. 1(1), 49-59. 